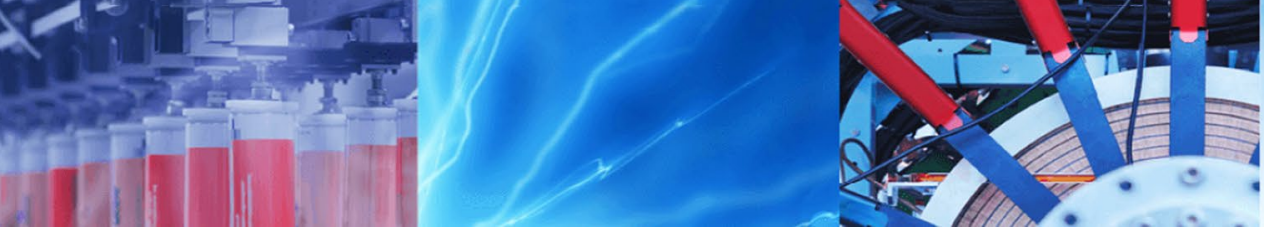

Research Article

\title{
Comprehensive analysis and synthesis of time delay compensated deadbeat control strategy
}

\author{
Rabia Nazir ${ }^{1}$ (D) Abida Nazir ${ }^{2} \cdot$ Aqsa Shabbir $^{3} \cdot$ Ali Hussain Kazim ${ }^{4}$
}

Received: 29 October 2019 / Accepted: 10 January 2020 / Published online: 24 January 2020

(c) Springer Nature Switzerland AG 2020

\begin{abstract}
This research comprehensively investigates the effect of uncertain time delay on performance of the deadbeat control. A solution based on a non-integer order Smith predictor has been proposed and analyzed to compensate uncertain time delays occurring in a stable closed loop system. Deadbeat control has been widely and conveniently used in the control of linear and non-linear systems. However, this control technique is severely sensitive to unmodeled dynamics and parameter uncertainties. Thus the performance of system may deviate significantly and hence lead to instability in practical cases. Time delay in a closed loop control system is a common source of unmodeled dynamics, which severely deteriorates control accuracy and results in reduced stability margin of the deadbeat control system. Physical system considered in this research work is a single-phase inverter. The simulation results indicate that the Smith predictor based deadbeat control scheme offers accurate time delay compensations and thus enhances the robustness and stability margin. Application example comprehensively illustrates effectiveness of the proposed control technique.
\end{abstract}

Keywords Deadbeat control $\cdot$ Smith predictor $\cdot$ Uncertain time delay $\cdot$ Single-phase converter

\section{Introduction}

Good dynamic properties of deadbeat control, like low steady-state error, quick rise and settling time, make it a convenient and simple choice in various process controls [1-6]. Deadbeat control technique in comparison to other modern control techniques (e.g resonant control, repetitive control) is simple, quick and achieves the steady state in minimum number of time steps. However, performance of deadbeat control is highly sensitive to parameter uncertainties and unmodelled dynamics of the system [7-10]. Unmodelled dynamics may cause unexpected delay in the systems. Uncertain time delays occur in control systems due to many reasons including processing delay of measuring and control circuits, unmodelled dynamics and non-linearities. Uncertain time delay in deadbeat controlled systems is a critical issue resulting in degraded performance $[9,11]$.

The issue of time delay compensation in deadbeat controlled converters has been considered but not thoroughly addressed. One way of compensating uncertain time delay is to include repetitive controller along with the deadbeat and then employ phase lead compensation [12]. However, phase lead compensation cannot be directly used with the deadbeat control alone due to the non-causal delay item $z^{n}$ where $n$ is the number of phase lead steps. Another simple compensation method is two step forward prediction approach proposed in the frame of model predictive control $[13,14]$. This approach only compensates for the computational delay of the controller whereas the uncertain delay

$\triangle$ Rabia Nazir, rabia.nazir@uet.edu.pk; Abida Nazir, anazir.pg@smme.edu.pk; Aqsa Shabbir, aqsa_shabbir@outlook.com; Ali Hussain Kazim, ali.h.kazim@uet.edu.pk | ${ }^{1}$ Electrical Engineering Department, University of Engineering and Technology (UET) Lahore, Lahore, Pakistan. ${ }^{2}$ Department of Bio-Medical Engineering and Sciences, National University of Science and Technology (NUST), Islamabad, Pakistan. ${ }^{3}$ Electrical Engineering Department, Lahore College for Women University (LCWU), Lahore, Pakistan. ${ }^{4}$ Mechanical Engineering Department, University of Engineering and Technology (UET) Lahore, Lahore, Pakistan. 
due to other factors cannot be effectively compensated. Therefore, there is a need for a unified approach to effectively compensate overall uncertain delays occurring in the control system.

The Smith Predictor is a model based controller design technique, where an estimate of the plant/process along with uncertain delay is included in the controller and delay is effectively compensated $[11,15-20]$. The conventional digital Smith predictor is an integer order controller employing a time delay element $z^{-N}$ where $N=T_{d} / T_{s}$ with $T_{d}$ being the uncertain time delay of the plant/process and $T_{s}$ being the sampling frequency of the digital Smith predictor. $T_{s}$ is usually fixed and it is impractical to vary the sampling frequency of the controller as it requires the redesigning and tuning of the controller parameters. Therefore, variable $T_{d}$ may result in non-integer order $N$ of the Smith predictor. The conventional Smith predictor can only realize integer delays so in case of non-integer $N$ order is rounded off to the nearest integer value which results in approximate delay compensation and hence the performance degrades. In this research work, a fractional-order Smith Predictor delay compensation approach has been proposed and used for a deadbeat controlled single phase inverter. Fractional-order Smith predictor proposed and implemented in this research paper is able to achieve better control accuracy and robustness performance, which effectively suggests its widespread applications in practical systems. This control technique overcomes the uncertain delays due to controller, plant, measurement and computational time occurring a feedback control systems. Detailed analysis and synthesis of the proposed control scheme have also been carried out and provided. Various time delay scenarios considered, demonstrate the feasibility and effectiveness of the proposed scheme.

\section{Smith predictor for an arbitrary time delay}

\subsection{Conventional Smith Predictor}

Smith predictor has proven to be an effective delay compensation solution. A conventional Smith predictor is shown in Fig. 1 , where $G_{p}(z)$ is the plant, $z^{-N}$ is the delay, $G_{p}(z)$ is the estimated plant model, $G_{c}(z)$ represents the deadbeat controller, $R(z)$ is input and $C(z)$ is output of the system.

Overall transfer function of the conventional Smith predictor shown in Fig. 1 is as follow.

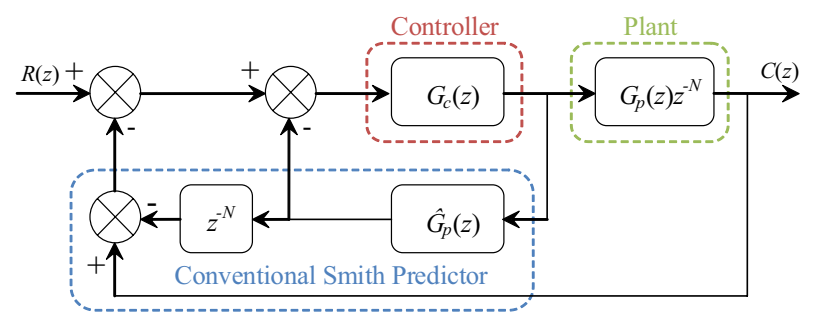

Fig. 1 Conventional Smith predictor based deadbeat control scheme

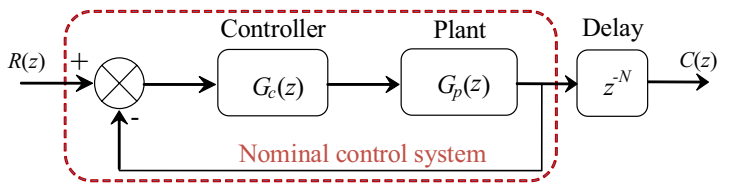

Fig. 2 A simplified block diagram of the control system shown in Fig 1

$\frac{C(z)}{R(z)}=\frac{G_{p}(z) G_{c}(z) z^{-N}}{1+G_{x}(z) G_{c}(z)+G_{p}(z) G_{c}(z) z^{-N}}$

where,

$G_{x}(z)=G_{p}(z)\left(1-z^{-N}\right)$

If the predicted/estimated model of the plant $G_{p}(z)$ is very accurate then $\hat{G_{p}} \hat{(z)}=G_{p}(z)$ otherwise $\hat{G_{p}}(z) \approx G_{p}(z)$ then (1) becomes,

$\frac{C(z)}{R(z)} \approx \frac{G_{p}(z) G_{c}(z) z^{-N}}{1+G_{p}(z) G_{c}(z)}$

Equation (3) represents that by the application of a Smith predictor, a plant $G_{p}(z)$ subjected to an uncertain time delay $z^{-N}$ in a closed loop, and controlled by a controller $G_{p}(z)$ becomes equivalent to a nominal closed loop system $\frac{f_{c}(z) G_{p}(z)}{1+G_{c}(z) G_{p}(z)}$ separated from the same time delay as shown in Fig. 2. By applying a Smith predictor, stability of the overall system is guaranteed if and only if, poles of the nominal closed loop system followed by time delay element exists inside a unit circle. Poles of the pure delay element always exist at the center of the unit circle thus the stability criteria is based on poles of the nominal closed loop transfer function only. 
In digital implementations, the time delay element $z^{-N}$ can only be realized with integer value $N$. However, in practical implementation $N=T_{d} / T_{s}$, where $T_{d}$ is the uncertain time delay of the system and $T_{s}$ is fixed sampling period, may result in non-integer value. Therefore, implementation of integer period, $N$, based Smith predictor in some applications restricts performance of the control system. In order to deal with this issue a Fractional Order Smith Predictor has been proposed, implemented and analyzed.

\subsection{Fractional order Smith predictor}

In the events of mismatch between $T_{d}$ and $T_{s}$, their ratio $T_{d} / T_{s}$ becomes non-integer which leads to sever frequency robustness issues in power converter applications. In such cases, there is a need to implement Smith predictor based controller with non-integer $\operatorname{order} N$. otherwise, performance of overall system degrades.

When the ratio of $T_{d}$ and $T_{s}$ is non-integer, assuming that the non-integer ratio $N$ can be expressed as $N=N_{i}+F$ where $N_{i}=\lfloor N\rfloor$ and $F=N-N_{i}$, the required delay can be realized as $z^{-N}=z^{-N_{i}-F}$. In other words, $N$ is divided into two components; an integer part $N_{i}$ and a fractional part $F$. The delay based on the fractional part $z^{-F}$ cannot be realized directly in digital implementations. Therefore, the fractional delay $z^{-F}$ can be approximated by employing a Lagrange interpolation based fractional delay filter as follow [21]:

$z^{-F} \approx \sum_{i=1}^{n} a_{i} z^{-i}$

where $a_{i}$ is the coefficients of each delay terms and approximation in the above equation can be converted to equality if $n$ is equal to infinity. Practically $n=\inf$ is not possible therefore $n$ is limited to some high value. However, value of $n$ directly effects computational cost and complexity. The coefficients $a_{i}$ of delay terms can be calculated as:

$a_{i}=\prod_{j=1, j \neq i}^{n} \frac{F-j}{i-j}, \quad i, j=1,2,3 \ldots n$

To demonstrate the accuracy of approximation of fractional delay $z^{-F}$ using Lagrange interpolations, the magnitude responses of the proposed scheme are shown in Fig. 3. It is noticed that increasing $n$ results in higher bandwidth at the cost of computational burden. Even a first order filter achieves a good approximation within 50\% of the Nyquist frequency. That is to say that the designed filter accurate;y compensates any arbitrary delay within this bandwidth. In practical applications, in order to keep a

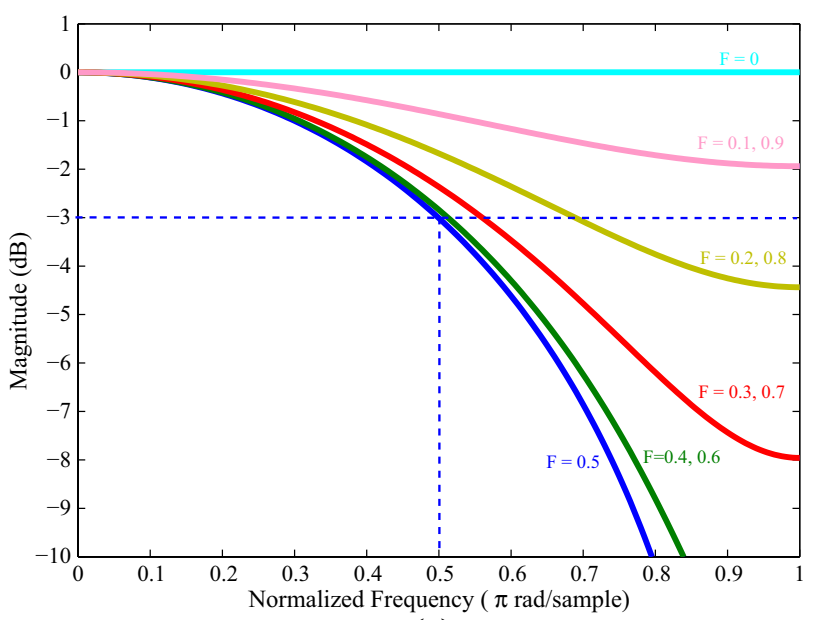

(a)

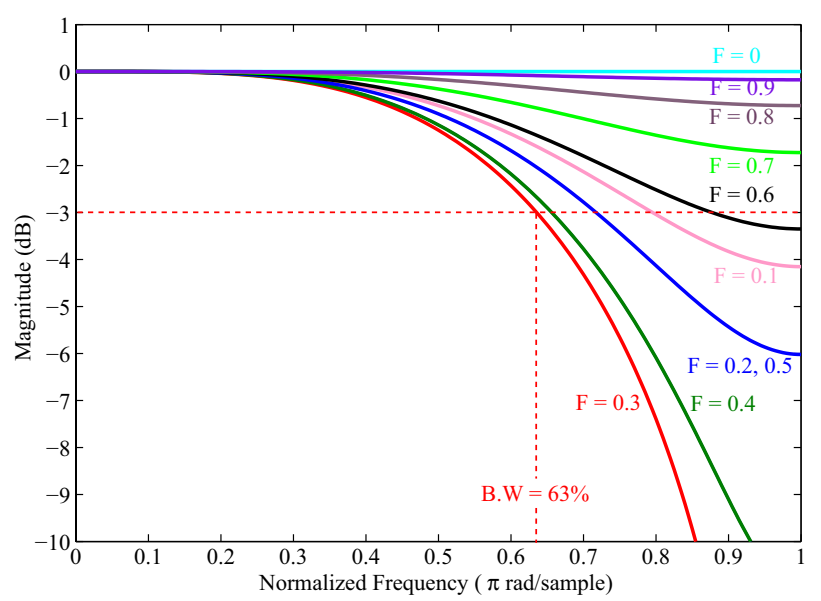

(b)

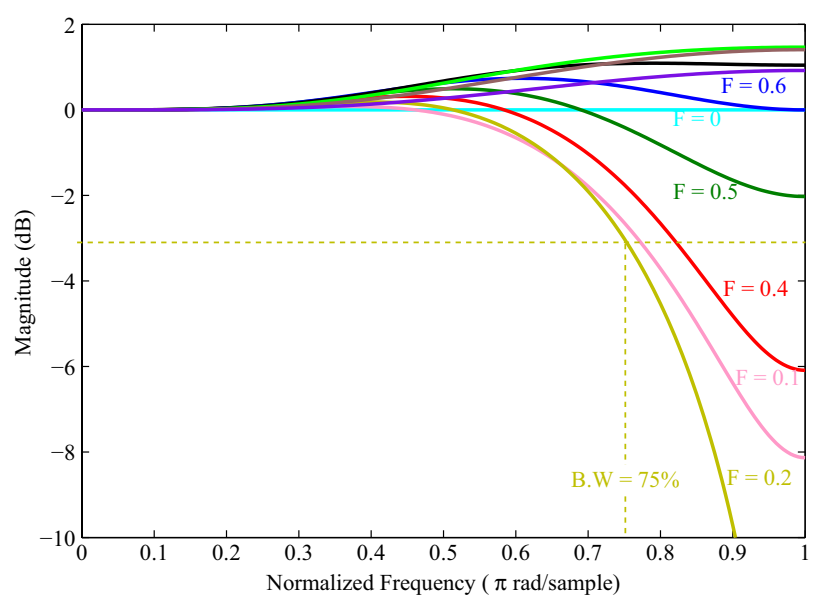

(c)

Fig. 3 Magnitude responses of the fractional delay filters a $n=1$, b $n=2, \mathbf{c} n=3$ 
reasonable balance between simplicity and approximation accuracy, the order $n$ is usually chosen as 1 or 2 .

In the absence of time delay $\mathrm{N}=0, \mathrm{Ni}=0, \mathrm{~F}=\mathrm{O}$ the $G_{x}(z)=G_{p}(z)$ and this (1) would reduce to the following:

$\frac{C(z)}{R(z)} \approx \frac{G_{c}(z) G_{p}(z)}{1+G_{c}(z) G_{p}(z)}$

Above equation implies that the proposed universal fractional-order Smith Predictor works as a unity subsystem and performance of the controller $G_{c}(z)$ would be no less than the conventional deadbeat control scheme.

\section{Fractional order Smith predictor based single-phase inverter}

\subsection{Modeling of single-phase PWM inverter}

The dynamics of a single-phase PWM inverter connected to a linear resistive load as shown in Fig. 4 can be represented as [22]:

$\left[\begin{array}{l}\dot{v}_{c} \\ \ddot{v}_{c}\end{array}\right]=\left[\begin{array}{cc}0 & 1 \\ -\frac{1}{L_{n} C_{n}} & -\frac{1}{R_{n} C_{n}}\end{array}\right]\left[\begin{array}{l}v_{c} \\ \dot{v}_{c}\end{array}\right]+\left[\begin{array}{c}0 \\ \frac{1}{L_{n} C_{n}}\end{array}\right] v_{a b}$

where, $v_{c}$ is output voltage, $v_{a b}$ is PWM modulated voltage available at the input of low pass $L C$ filter; $L_{n}, C_{n}$ and $R_{n}$ are nominal values of passive components. The system given by (7) is a linear system of the from $\dot{x}=A x+B u$. Its sampled data form can be achieved by applying a PWM pulse to the converter and thus $x((k+1) T) \approx e^{A T} x(k T)+e^{A T / 2} B E_{n} A T$ which after few manipulations becomes [21].

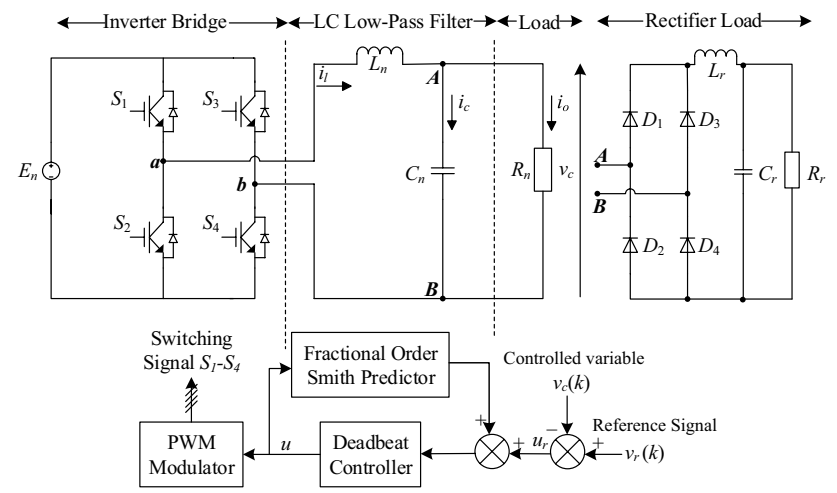

Fig. 4 Delay compensated deadbeat control of single-phasePWM inverter

SN Applied Sciences

$$
\begin{aligned}
{\left[\begin{array}{l}
v_{c}(k+1) \\
\dot{v}_{c}(k+1)
\end{array}\right] } & =\left[\begin{array}{ll}
\varphi_{11} & \varphi_{12} \\
\varphi_{21} & \varphi_{22}
\end{array}\right]\left[\begin{array}{l}
v_{c}(k) \\
\dot{v}_{c}(k)
\end{array}\right]+\left[\begin{array}{l}
g 1 \\
g 2
\end{array}\right] \Delta T(k) \\
y & =\left[\begin{array}{ll}
1 & 0
\end{array}\right]\left[\begin{array}{l}
v_{c}(k) \\
\dot{v}_{c}(k)
\end{array}\right]
\end{aligned}
$$

where

$$
\begin{aligned}
g_{1} & =T^{2} / 2 L_{n} C_{n} \\
g_{2} & =T / L_{n} C_{n}-T^{2} / 2 L_{n} C_{n}^{2} R_{n} \\
\varphi_{11} & =1-T^{2} / 2 C_{n} L_{n} \\
\varphi_{12} & =T / 3 C_{n}-T^{2} / 6 R_{n} C_{n}^{2} \\
\varphi_{21} & =-T / L_{n} C_{n}+T^{2} / 2 L_{n} C_{n}^{2} R_{n} \\
\varphi_{22} & =1-T / R_{n} C_{n}-T^{2} / 2 L_{n} C_{n}+T^{2} / 2 R_{n}^{2} C_{n}^{2}
\end{aligned}
$$

$v_{c}(k), \dot{v}_{c}(k)$ and $\Delta T(k)$ represent values of variables at sampling instant $t=k T$. The main control objective is to enable the output voltage $\left(v_{c}\right)$ to precisely and accurately track the ac reference signal $\left(v_{r}\right)$ in the presence of uncertain time delays.

\subsection{Controller design}

Controller for the single-phase PWM inverter shown in Fig. 4 is composed of two components: a plug-in fractional order Smith predictor and a conventional deadbeat controller.

\subsubsection{Conventional deadbeat controller}

The conventional deadbeat controller for a plant described by (8) can be achieved by calculating its Auto Regressive Moving Average (ARMA) equation and then replacing $u(k+1)$ by $v_{r}(k)$ as the deadbeat control achieves tracking of the signal in next time step, that is to say $H(z)=z^{-1}$. Thus the control signal $u(k)$ is:

$u(k)=\frac{1}{m_{1}}\left(v_{r}(k)+p_{1} y(k)-m_{2} u(k-1)+p_{2} y(k-1)\right)$

where, $u(k)= \pm \Delta T(k), \quad m_{1}=g_{1}, m_{2}=\varphi_{12} g_{2}-\varphi_{22} g_{1}$, $p_{1}=-\varphi_{22}-\varphi_{11} p_{2}=\varphi_{22} \varphi_{11}-\varphi_{21} \varphi_{12}$, and $v_{r}(k)=y(k+1)$.

\subsubsection{Fractional Order Smith Predictor}

Practically, the performance of conventional deadbeat controller undergoes severe fluctuations due to unmodeled dynamics and parameter uncertainties including uncertain time delay. Therefore, a fractional order Smith predictor can be plugged into the deadbeat controlled 


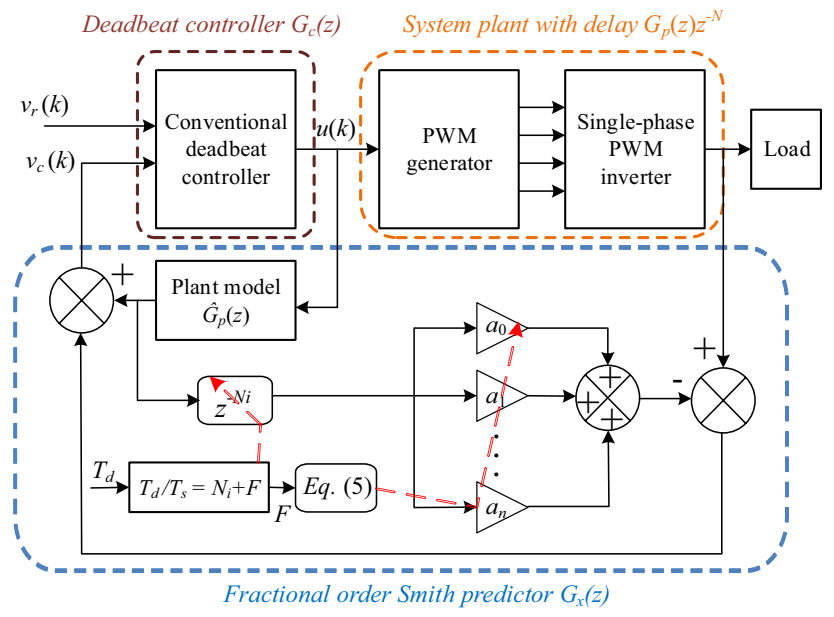

Fig. 5 Fractional-order Smith predictor based deadbeatcontrol scheme for single-phase PWM inverter

inverter to overcome performance degradation issues due to uncertain time delay, as shown in Fig. 5.

\subsection{Simulation Results and Discussion}

Performance of the proposed control scheme is tested and evaluated on a single-phase PWM inverter system. Nominal values of the converter and control parameters are given in Table 1.Figure 6 shows response of only deadbeat controlled single-phase inverter with rectifier load under three different time delays $T_{d}=0,1 e^{-4}, 2 e^{-4}$ in (a), (b) and (d) respectively. It is clear that deadbeat control can only track the reference signal in the absence of time delay. Thus, it can be concluded that the performance of the deadbeat control degrades drastically in the presence of uncertain time delay. Figure $6 c$, $d$ represent very high tracking error in the presence of time delay. Long time delay leads to higher error and thus the system may
Table 1 Inverter system parameters

\begin{tabular}{lll}
\hline Parameters & Symbol & Value \\
\hline Input dc voltage & $E_{n}$ & $120 \mathrm{~V}$ \\
Load resistance & $R_{r}$ & $30 \Omega$ \\
Load inductance & $L_{r}$ & $5 \mathrm{mH}$ \\
Load capacitance & $C_{r}$ & $500 \mu \mathrm{F}$ \\
Output voltage (rms) & $v_{c}$ & $40 \mathrm{~V}$ \\
Sampling frequency & $f_{s}$ & $10 \mathrm{kHz}$ \\
Sampling time & $T_{s}$ & $1 e^{-4} \mathrm{~s}$ \\
Filter inductance (Low-pass) & $L_{n}$ & $5 \mathrm{mH}$ \\
Filter capacitance (Low-pass) & $C_{n}$ & $60 \mu \mathrm{F}$ \\
Fractional delay filter order & $n$ & 2 \\
Nominal fundamental frequency & $f$ & $50 \mathrm{~Hz}$ \\
\hline
\end{tabular}

become unstable in the absence of time delay compensation techniques.

Figure 7 shows the response of fractional order Smith predictor based deadbeat controlled inverter for two different non-integer time delays $\left(T_{d}=1.2 e^{-4}, 2.3 e^{-4}\right)$. A fractional order Smith predictor having $n=2$ has been employed. It is clear from Fig. $7 a, b$ that the output of inverter tracks the reference voltage with acceptable/low steady-state error and Total Harmonic Distortion (THD) much less than $1 \%$. In order to further emphasize the significance of fractional order Smith predictor based deadbeat control it has been compared with the conventional Smith predictor based control technique. Figure 8 shows the response of conventional Smith predictor based compensation technique when delay time is $T_{d}=3.5 e^{-4}$. Since, the conventional Smith predictor can only compensate integer delay therefore a delay of $3.5 e^{-4}$ is rounded off to the nearest integers 3, 4 and an increase in steady-state error and THD has been observed in both cases. THD is greater than $1 \%$ because $N=3,4$ cannot compensate the 


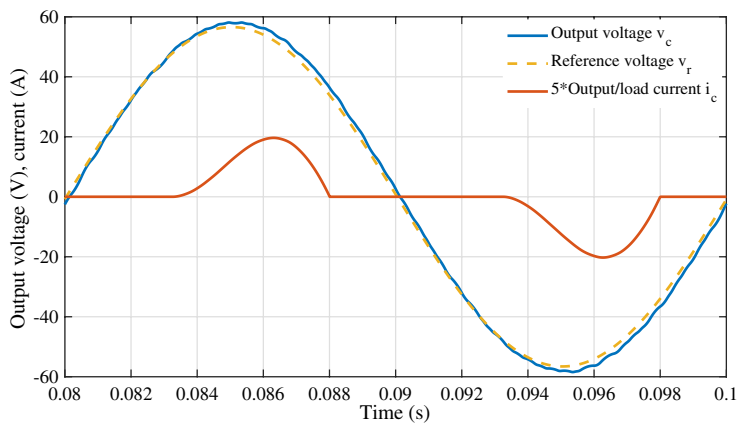

(a) Steady-state response of deadbeat controlled inverter.

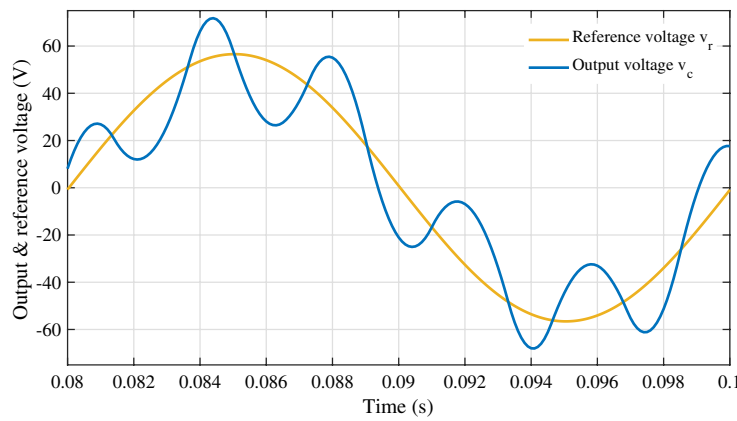

(b) Deadbeat controlled inverter with time delay $T_{d}=1 e^{-4}$.

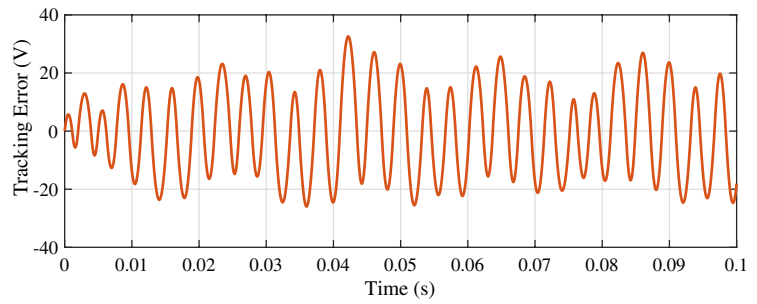

(c) Tracking error with time delay $T_{d}=1 e^{-4}$.

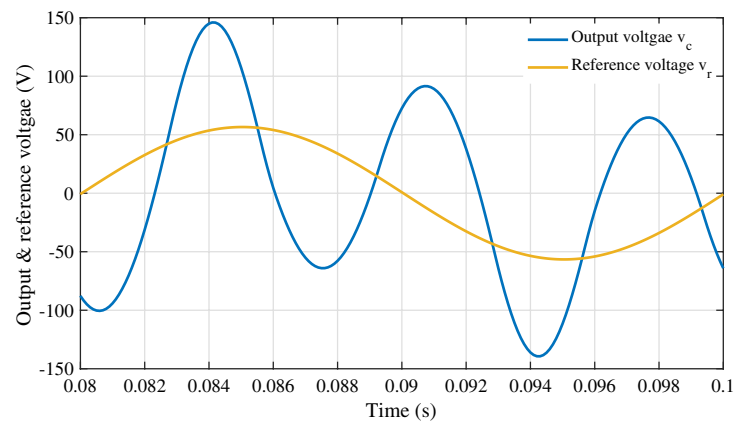

(d) Deadbeat controlled inverter with time delay $T_{d}=2 e^{-4}$.

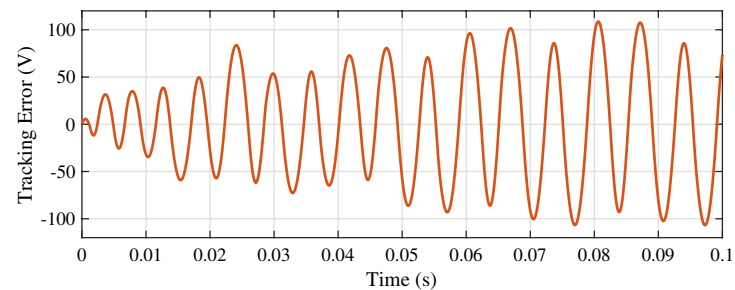

(e) Tracking error with time delay $T_{d}=2 e^{-4}$.

Fig. 6 Steady-state response of deadbeat controlled inverter with rectifier load under various time delays

SN Applied Sciences

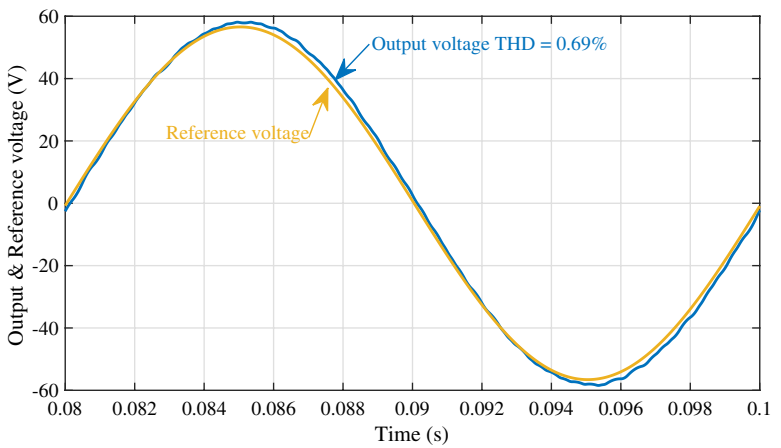

(a) Steady-state response when time delay $T_{d}=1.2 e^{-4}$.

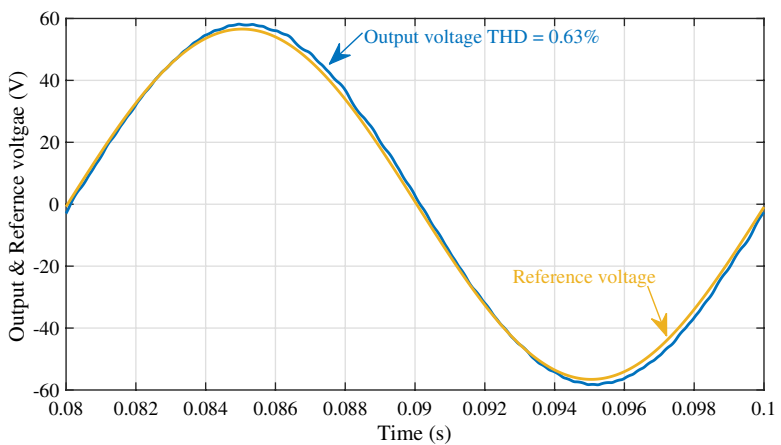

(b) Steady-state response when time delay $T_{d}=2.3 e^{-4}$.

Fig. 7 Steady-state response of deadbeat control along with fractional order Smith predictor under various time delays

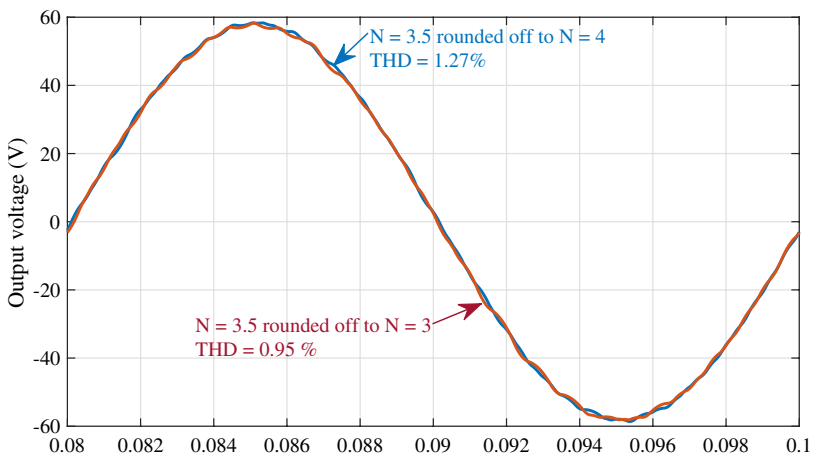

Fig. 8 Steady-state response of deadbeat control along with conventional Smith predictor when time delay $T_{d}=3.5 e^{-4}$

required delay accurately hence the need of a fractional delay compensation technique is clear.

Table 2 compares the performance of three different control techniques. Techniques compared are deadbeat control, conventional Smith predictor based deadbeat control (Deadbeat+SP) and fractional order Smith predictor based deadbeat control (Deadbeat+FOSP). Conventional deadbeat control can only be implemented with integer delays, therefore, performance isn't evaluated where fractional delay occurs. For conventional Smith predictor based deadbeat control the delays are approximated to 
Table 2 Comparison of control techniques

\begin{tabular}{|c|c|c|c|c|c|c|}
\hline \multirow[t]{2}{*}{ Delay } & \multicolumn{2}{|l|}{ Deadbeat } & \multicolumn{2}{|c|}{ Deadbeat + SP } & \multicolumn{2}{|c|}{ Deadbeat + FOSP } \\
\hline & Error (V) & THD\% & Error (V) & THD\% & Error (V) & THD\% \\
\hline 1 & 30 & 44 & 3.2 & 0.69 & 3.2 & 0.69 \\
\hline 1.2 & - & - & 4.8 & 1.8 & 3.6 & 0.83 \\
\hline 2 & 110 & 278.6 & 3 & 0.63 & 3 & 0.63 \\
\hline 2.3 & - & - & 4.9 & 1.5 & 4.1 & 0.69 \\
\hline 3 & 191.7 & 381.7 & 3.6 & 0.65 & 3.6 & 0.65 \\
\hline 3.5 & - & - & 5.8 & 1.27 & 3.9 & 0.75 \\
\hline 4 & 265 & 370.7 & 4 & 0.7 & 4 & 0.7 \\
\hline 4.6 & - & - & 5.1 & 1.02 & 3.6 & 0.89 \\
\hline 5 & 353.9 & 495.4 & 3.5 & 0.64 & 3.5 & 0.64 \\
\hline
\end{tabular}

the nearest integer value and then performance is evaluated and it can be seen that where the required delay is non-integer approximation results in increased steadystate error and THD. For fractional order Smith predictor based deadbeat control the THD is always lower than $1 \%$ for the evaluated delay cases. When the required delay is integer Deadbeat+FOSP becomes exactly same as that of Deadbeat+SP and hence the performance is similar.

\section{Conclusion}

This paper develops and applies a fractional-order Smith Predictor based delay compensation technique, for output ac voltage control of a single-phase PWM inverter when arbitrary time delays occur in the control system. Using this technique any arbitrary time delay occurring in the deadbeat controlled system can be accurately approximated and compensated. A deadbeat control scheme which is capable of compensating unknown time delays accurately and effectively promotes its widespread applications in practical systems. Simulated inverter system demonstrates the feasibility of proposed technique. Results indicate high tracking accuracy, fast transient response, and good adaptation to uncertain delay time. The proposed simple and effective fractional-order time delay compensation solution can be applied with minor changes to other applications including unmanned aerial vehicles and predictive control.

\section{Compliance with ethical standards}

Conflict of interest The authors declare that they have no conflict of interest.

\section{References}

1. Verschueren R, Ferreau HJ, Zanarini A, Mercangöz M, Diehl M (2017) A stabilizing nonlinear model predictive control scheme for time-optimal point-to-point motions. In: 2017 IEEE 56th annual conference on decision and control (CDC), pp 2525-2530

2. Ne D, Mareels I, Bastin G, Mahony RE (1998) Output dead beat control for a class of planar polynomial systems. SIAM J Control Optim 36(1):253-272

3. Tuna SE (2012) State deadbeat control of nonlinear systems: construction via sets. Automatica 48(9):2201-2206

4. Gokhale KP, Kawamura A, Hoft RG (1987) Dead beat microprocessor control of PWM inverter for sinusoidal output waveform synthesis. IEEE Trans Ind Appl 23(5):901-910

5. Wen Q, Zhu L, Fu C, Research on deadbeat current control strategy of three-phase PWM voltage source rectifier. In: Proceedings of the 2nd international conference on computer science and electronics engineering, $\mathrm{pp}$ 1769-1772

6. Kawabata T, Miyashita T, Yamamoto Y (1990) Dead beat control of three phase PWM inverter. IEEE Trans Power Electron $5(1): 21-28$

7. Xing X, Zhang Z, Zhang C, He J, Chen A (2017) Space vector modulation for circulating current suppression using deadbeat control strategy in parallel three-level neutral-clamped inverters. IEEE Trans Ind Electron 64(2):977-987

8. Xing X, Zhang C, Chen A, Geng H, Qin C (2018) Deadbeat control strategy for circulating current suppression in multiparalleled three-level inverters. IEEE Trans Ind Electron 65(8):6239-6249

9. Mattavelli $P$ (2005) An improved deadbeat control for ups using disturbance observers. IEEE Trans Ind Electron 52(1):206-212

10. Nazir R (2017) Taylor series expansion based repetitive controllers for power converters, subject to fractional delays. Control Eng Pract 64:140-147

11. Richard JP (2003) Time-delay systems: an overview of some recent advances and open problems. Automatica 39(10):1667-1694

12. Zhang B, Wang D, Zhou K, Wang Y (2008) Linear phase lead compensation repetitive control of a CVCF PWM inverter. IEEE Trans Ind Electron 55(4):1595-1602

13. Young HA, Perez MA, Rodriguez J (2016) Analysis of finitecontrolset model predictive current control with model parameter mismatch in a three-phase inverter. IEEE Trans Ind Electron 63(5):3100-3107

14. Vazquez S, Rodriguez J, Rivera M, Franquelo LG, Norambuena M (2017) Model predictive control for power converters and drives: advances and trends. IEEE Trans Ind Electron 64(2):935-947

15. Bosio FD, Ribeiro LADS, Freijedo FD, Pastorelli M, Guerrero JM (2017) Discrete-time domain modeling of voltage source 
inverters in standalone applications: enhancement of regulators performance by means of Smith Predictor. IEEE Trans Power Electron 32(10):8100-8114

16. Lai CL, Hsu PL (2010) Design the remote control system with the time-delay estimator and the adaptive smith predictor. IEEE Trans Ind Electron 6(1):73-80

17. Stojic MR, Matijevic FS, Draganovic LS (2001) A robust Smith predictor modified by internal models for integrating process with dead time. IEEE Trans Autom Control 46(8):1293-1298

18. Lu M, Wang X, Loh PC, Blaabjerg F, Dragicevic T (2018) Graphical evaluation of time-delay compensation techniques for digitally controlled converters. IEEE Trans Power Electron 33(3):2601-2614

19. Liu T, Hao S, Li D, Chen W, Wang Q (2019) Predictor-based disturbance rejection control for sampled systems with input delay. IEEE Trans Control Syst Technol 27(2):772-780
20. Sakthivel R, Mohanapriya S, Ahn CK, Karimi HR (2019) Output tracking control for fractional-order positive switched systems with input time delay. IEEE Trans Circuits Syst II Express Briefs 66(6):1013-1017

21. Nazir R (2015) Advanced repetitive control of grid converters for power quality improvement, Phd Thesis,

22. Wang Z, Zhou K, Li S, Yang Y (Nov 2018) Fractional-order time delay compensation in deadbeat control for power converters. In: 2018 IEEE international power electronics and application conference and exposition (PEAC), pp 1-6

Publisher's Note Springer Nature remains neutral with regard to jurisdictional claims in published maps and institutional affiliations. 